\title{
Justificam-se os indicadores de contratualização dos cuidados de saúde primários sobre rastreios oncológicos?
}

Bruno Heleno*

4 remuneração associada a indicadores de desempenho é um incentivo poderoso para a mudança de comportamentos de profissionais de saúde. Existem poucos estudos de elevada qualidade sobre o assunto, mas o pagamento por desempenho em cuidados primários parece estar associado a pequenas melhorias na qualidade dos cuidados de saúde prestados. ${ }^{1-3}$ Neste editorial procurar-se-á refletir sobre qual a redução de mortalidade esperada com os programas de rastreio oncológico incentivados pelos indicadores de desempenho, quais os principais malefícios que podem advir destes rastreios e como os indicadores criam conflitos de interesse para os médicos de família.

\section{QUAL A REDUÇÃO DE MORTES QUE PODE SER ESPERADA PELO RASTREIO DE CANCRO?}

Os rastreios de cancro têm como objetivo principal reduzir o número de mortes por cancro. No princípio de 2015 foi publicado um artigo importantíssimo que reviu 48 ensaios clínicos e 9 meta-análises de ensaios clínicos sobre rastreio. ${ }^{4}$ As conclusões devem fazer-nos pensar: "Entre os testes de rastreio (para doenças onde a morte é uma ocorrência frequente) atualmente disponíveis, reduções de mortalidade específica são incomuns e reduções de mortalidade global são muito raras ou não existentes" (tradução do autor). Apenas três dos dez programas de rastreio avaliados (rastreio com mamografia, rastreio com pesquisa de sangue oculto nas fezes e rastreio com sigmoidoscopia) estão associados a reduções do número de mortes pelo cancro rastreado.

* Departamento de Medicina Geral e Familiar, NOVA Medical School/Faculdade de Ciências Médicas, Universidade Nova de Lisboa
O Quadro mostra a redução esperada do número de cancros com os rastreios que fazem parte do painel de indicadores de contratualização dos cuidados de saúde primários. Os dados são baseados nas revisões sistemáticas publicadas pela colaboração Cochrane. ${ }^{5-6} \mathrm{O}$ rastreio do cancro do colo do útero nunca foi avaliado em ensaios clínicos, mas foi implementado, uma vez que estudos observacionais mostraram reduções dramáticas (RRR entre 60-90\%) na incidência por este cancro. ${ }^{7}$ Os dados mostram que mesmo para os cancros em que há reduções significativas de mortalidade, essas reduções são relativamente modestas (1-2 mortes evitadas por 1.000 pessoas rastreadas durante 10 anos). Divulgar estes números é importante: existem estudos que mostram que doentes e médicos sobrestimam o número de vidas salvas pelo rastreio. ${ }^{8-10}$

\section{E HÁ PROBLEMAS IMPORTANTES COM OS RASTREIOS?}

A designação "cancro" inclui um largo espetro de lesões, desde aquelas que serão sempre mortais se não forem tratadas (ou mesmo mortais, ainda que tratadas) até lesões indolentes com um potencial extremamente baixo de progressão metastática e morte. ${ }^{11} \mathrm{Na}$ ausência de rastreio, os médicos apenas conseguem detetar os cancros que causam sintomas e, consequentemente, aqueles que virão a tornar-se fatais se não tratados. Dado que parte destes cancros tem mau prognóstico quando já evoluiu para sintomas, surgiu a ideia de aplicar testes em pessoas sem sintomas para procurar detetar lesões mais pequenas, pré-clínicas, na expectativa de melhorar o seu prognóstico. ${ }^{12} \mathrm{O}$ reverso da medalha é que detetam-se não só os cancros fatais como um conjunto de lesões indolentes. O dramático 


\begin{tabular}{|c|c|c|c|c|}
\hline \multicolumn{5}{|l|}{ QUADRO } \\
\hline Intervenção & Referência & $\begin{array}{l}\text { Redução relativa do } \\
\text { risco de morte por } \\
\text { cancro (intervalo de } \\
\text { confiança } 95 \% \text { ) }\end{array}$ & $\begin{array}{l}\text { Número de mortes } \\
\text { esperado numa } \\
\text { população não } \\
\text { rastreada* }\end{array}$ & $\begin{array}{c}\text { Número de vidas } \\
\text { salvas atribuíveis ao } \\
\text { rastreio }\end{array}$ \\
\hline $\begin{array}{l}\text { Rastreio do cancro da } \\
\text { mama com mamografia }\end{array}$ & $\begin{array}{l}\text { Gøtzsche and } \\
\text { Jørgensen, } 2013^{5} \\
\text { (todos os ensaios) } \dagger\end{array}$ & RRR 19\% (13\%-26\%) & 5 por 1.000 & 1 por 1.000 \\
\hline $\begin{array}{l}\text { Rastreio do cancro } \\
\text { colorretal com pesquisa } \\
\text { de sangue oculto }\end{array}$ & Holme et al, 20136 & RRR 14\% (8\%-20\%) & 8 por 1.000 & 1 por 1.000 \\
\hline $\begin{array}{l}\text { Rastreio do cancro } \\
\text { colorretal com } \\
\text { sigmoidoscopia }\end{array}$ & Holme et al, 20136 & RRR 28\% (21\%-35\%) & 8 por 1.000 & 2 por 1.000 \\
\hline $\begin{array}{l}\text { Rastreio do cancro do } \\
\text { colo do útero com } \\
\text { colpocitologia }\end{array}$ & $\begin{array}{l}\text { Nunca avaliado em } \\
\text { ensaios clínicos }\end{array}$ & ND & ND & ND \\
\hline
\end{tabular}

RRR: redução do risco relativo; ND: não disponível.

* Mediana da mortalidade específica encontrada no grupo de controlo dos estudos incluídos na meta-análise; † Esta é a meta-análise mais otimista dos efeitos do rastreio com mamografia. Os autores da meta-análise argumentam que nos devíamos basear apenas nos ensaios clínicos de melhor qualidade. Assim sendo, o RR de morte por cancro da mama com rastreio é $0,90(0,79-1,02)$ e a diferença de risco absoluto é 0 por 1000 .

da situação é que, com a tecnologia que temos habitualmente disponível (anatomia patológica, citoquímica, marcadores imunológicos, etc.), não conseguimos distinguir entre cancros fatais e indolentes. Por isso, acabamos por tratar de forma agressiva todas as lesões. ${ }^{13-15}$

Os doentes que têm cancros que virão a ser fatais podem vir a beneficiar desta deteção precoce. Mas os doentes com cancros indolentes, que nunca viriam a ter sintomas do cancro, só saem prejudicados por este tratamento agressivo e não têm qualquer possibilidade de ter benefício. Por isso, dizemos que estes doentes foram "sobrediagnosticados" e "sobretratados". Tanto o diagnóstico como o tratamento não podiam melhorar o prognóstico de uma doença que nunca viria a evoluir, ou para sintomas ou para morte.

\section{DECISÃO INFORMADA E CONSENTIMENTO INFORMADO}

Sempre que estão em jogo aspetos importantes da saúde dos utentes e existe uma grande incerteza se os benefícios de uma intervenção ultrapassam os seus malefícios é importante procurar a decisão partilhada e o consentimento informado. ${ }^{16}$ Logo, a decisão partilhada e o consentimento informado são fundamentais quando os médicos de família convidam os seus utentes para o rastreio. Tanto mais que a medicina geral $\mathrm{e}$ familiar se orgulha de ser uma especialidade que pratica o método centrado na pessoa ${ }^{17} \mathrm{e}$ valoriza um modelo de concordância terapêutica face a um modelo de simples adesão do doente ao plano de tratamento.

Atualmente os indicadores de contratualização avaliam a proporção de utentes com determinado rastreio registado no processo. Ou seja, a tónica não está em fomentar a decisão partilhada face a intervenções com benefícios modestos e potenciais malefícios, mas antes em encorajar a adesão dos utentes a esta intervenção. Para além disso, no caso da mamografia há um incentivo financeiro para que seja oferecida uma intervenção que é ferozmente discutida no meio científico. ${ }^{5,18-19}$ Entre cumprir o indicador de contratualização e manter-se firme na defesa dos melhores interesses 
do utente, os médicos de família escolherão conscientemente a segunda. Mas o conflito de interesses era desnecessário. Por isso, estes indicadores são desadequados do ponto de vista ético e chegam a ser contrários à própria natureza da especialidade porque desencorajam a tomada de decisão partilhada.

\section{CONCLUSÃO}

Em resumo, se a remuneração associada ao desempenho é um mecanismo poderoso ao direcionar a atenção dos médicos para determinadas intervenções em saúde, ela deveria ser reservada para situações em que os benefícios ultrapassam largamente os malefícios. Como o benefício dos rastreios em termos de mortalidade é modesto e existe prejuízo para alguns dos participantes sob a forma de sobrediagnóstico de cancro, os indicadores associados a programas de rastreio não se justificam. Adicionalmente, com a formulação atual, estes indicadores estimulam o comportamento errado. Quando estamos perante situações em que há grande incerteza se os benefícios ultrapassam os malefícios, o que deveríamos incentivar é a tomada de decisão partilhada e não a adesão cega a determinada intervenção. Isto significa que é altura de substituir estes indicadores do conjunto daqueles que são contratualizados com as USF e UCSP.

\section{REFERÊNCIAS BIBLIOGRÁFICAS}

1. Scott A, Sivey P, Ait Ouakrim D, Willenberg L, Naccarella L, Furler J, et al. The effect of financial incentives on the quality of health care provided by primary care physicians. Cochrane Database Syst Rev. 2011;9: CD008451.

2. Melo M, de Sousa JC. Os indicadores de desempenho contratualizados com as USF: um ponto da situação no actual momento da reforma [Performance indicators contracted with family health units: a progress report on the current moment of primary health care reform in Portugal]. Rev Port Clin Geral. 2011;27(1):28-34. Portuguese

3. Santos I, Ribeiro IL. Indicadores de desempenho na consulta. Rev Port Clin Geral. 2009;25(2):228-36.

4. Saquib N, Saquib J, loannidis JP. Does screening for disease save lives in asymptomatic adults? Systematic review of meta-analyses and randomized trials. Int J Epidemiol. 2015;44(1):264-77.

5. Gøtzsche PC, Jørgensen KJ. Screening for breast cancer with mammography. Cochrane Database Syst Rev. 2013;6:CD001877.
6. Holme $\varnothing$, Bretthauer $M$, Fretheim A, Odgaard-Jensen J, Hoff G. Flexible sigmoidoscopy versus faecal occult blood testing for colorectal cancer screening in asymptomatic individuals. Cochrane Database Syst Rev. 2013;(9):CD009259.

7. Screening for squamous cervical cancer: duration of low risk after negative results of cervical cytology and its implication for screening policies: IARC Working Group on evaluation of cervical cancer screening programmes. Br Med J (Clin Res Ed). 1986;293(6548):659-64.

8. Hoffmann TC, Del Mar C. Patients' expectations of the benefits and harms of treatments, screening, and tests: a systematic review. JAMA Intern Med. 2015;175(2):274-86.

9. Wegwarth O, Schwartz LM, Woloshin S, Gaissmaier W, Gigerenzer G. Do physicians understand cancer screening statistics? A national survey of primary care physicians in the United States. Ann Intern Med. 2012;156(5):340-9.

10. Rodrigues R, Maria AR, Bragança A, Simões S, Tomé A, Rodrigues D, et al. Comunicação e percepção de risco: diferentes modos de comunicar, diferentes modos de partilhar a decisão clínica [Risk communication and risk perception: different ways of communicating, different ways of sharing clinical decisions]. Rev Port Med Geral Fam. 2015;31(2): 125-33. Portuguese

11. Esserman LJ, Thompson IM, Reid B, Nelson P, Ransohoff DF, Welch HG, et al. Addressing overdiagnosis and overtreatment in cancer: a prescription for change. Lancet Oncol. 2014;15(6):e234-42.

12. Raffle AE, Gray JA. Screening: evidence and practice. Oxford: Oxford University Press; 2007. ISBN 9780199214495

13. Brodersen J, Schwartz LM, Woloshin S. Overdiagnosis: how cancer screening can turn indolent pathology into illness. APMIS. 2014;122(8): 683-9.

14. Marcus PM, Prorok PC, Miller AB, DeVoto EJ, Kramer BS. Conceptualizing overdiagnosis in cancer screening. J Natl Cancer Inst. 2015 Apr; 107(4). doi: 10.1093/jnci/djv014

15. Welch HG, Black WC. Overdiagnosis in cancer. J Natl Cancer Inst. 2010;102(9):605-13.

16. Whitney SN, McGuire AL, McCullough LB. A typology of shared decision making, informed consent, and simple consent. Ann Intern Med. 2004; $140(1): 54-9$.

17. WONCA Europe. The European definition of general practice/family medicine. Barcelona: WONCA Europe; 2011.

18. Richards M.An independent review is under way. BMJ. 2011;343:d6843.

19. Marmot MG, Altman DG, Cameron DA, Dewar JA, Thompson SG, Wil$\operatorname{cox} M$. The benefits and harms of breast cancer screening: an independent review. $\mathrm{Br}$ J Cancer. 2013;108(11):2205-40.

\section{CONFLITO DE INTERESSES}

O autor declara não ter conflitos de interesses.

\section{ENDEREÇO PARA CORRESPONDÊNCIA}

bruno.heleno@fcm.unl.pt 